3. Ebenfalls ohne Erfolg macht die Beklagte geltend, das Eigentum an der Plakatsammlung sei dadurch, dass der Vater des Klägers diese nicht zur Rückerstattung angemeldet habe, kraft Gesetzes auf deren damaligen Besitzer übergegangen. Die Rückerstattungsanordnung hatte den Zweck, die beschleunigte Rückerstattung feststellbarer Vermögensgegenstände sicherzustellen (vgl. BGH Beschluss vom 28. Februar 1955 - GSZ 4/54, BGHZ 16, 350 [360]).

Konnten die durch die Anordnung begründeten Ansprüche aufgrund des Ausschlusscharakters der Anmeldefrist für sie nicht mehr durchgesetzt werden, musste derjenige, der den Gegenstand damals im Besitz hatte, zwar nicht mehr damit rechnen, Rückerstattungsansprüchen ausgesetzt zu sein. Ein originärer Eigentumserwerb durch Rückerstattungspflichtige, die lediglich den Besitz, nicht aber das Eigentum an dem entzogenen Gegenstand erlangt hatten, war hiermit aber nicht verbunden.
4. Gegen die Annahme des Berufungsgerichts, der Vater des Klägers habe das Eigentum an der Plakatsammlung auch nicht zu einem späteren Zeitpunkt eingebüßt, erhebt die Beklagte keine Einwände. Rechtsfehler sind insoweit auch nicht ersichtlich.

\section{IV.}

Das Berufungsurteil ist somit in dem durch die Revision angefochtenen Umfang aufzuheben (§562 Abs. 1 ZPO). Der Senat hat in der Sache selbst zu entscheiden, weil die Aufhebung des Urteils nur wegen einer Rechtsverletzung bei der Anwendung des Gesetzes erfolgt und nach letzterem die Sache zur Endentscheidung reif ist (§563 Abs. 3 ZPO).

V.

Die Kostenentscheidung beruht auf § 91 Abs. 1, § 92 Abs. 2, § 97 Abs. 1 ZPO. (Entscheidung von der Redaktion bearbeitet.)

\title{
Beweislastverteilung bei vermögensrechtlichen Rückgabeansprüchen
}

Bundesverwaltungsgericht, Beschluss vom 7. März 2012 - 5 B 56/11

\section{Zu Fragen der Beweislast und möglichen Beweiserleichterungen in einem Rechtsstreit um die Rückgabe eines Bildes nach § 5 Abs. 1 Satz 1 AusglLeistG. (Leitsatz der Redaktion)}

\section{Tenor}

- Die Beschwerde der Kläger gegen die Nichtzulassung der Revision in dem Urteil des Verwaltungsgerichts Gera vom 21. Juni 2011 wird zurückgewiesen.

Die Kläger tragen die Kosten des Beschwerdeverfahrens mit Ausnahme der außergerichtlichen Kosten der Beigeladenen.

Der Wert des Streitgegenstandes für das Beschwerdeverfahren wird auf 5.000,- EUR festgesetzt.

\section{Gründe}

Die Beschwerde hat keinen Erfolg. Die geltend gemachten Zulassungsgründe des $\S 132 \mathrm{Abs} .2 \mathrm{Nr}$. 1 und 3 VwGO liegen nicht vor.

1. Die Zulassung der Revision ist nicht wegen grundsätzlicher Bedeutung der angesprochenen Beweisfragen geboten ( $§ 32$ Abs. 2 Nr. 1 VwGO). Grundsätzliche Bedeutung kommt einer Rechtssache zu, wenn für die angefochtene Entscheidung der Vorinstanz eine konkrete, fallübergreifende und bislang höchstrichterlich ungeklärte Rechtsfrage des revisiblen Rechts von Bedeutung war, deren Klärung im Revisionsverfahren zu erwarten und zur Erhaltung der Einheitlichkeit der Rechtsprechung oder zur Weiterentwicklung des Rechts geboten ist (st. Rspr., vgl. z.B. Beschluss vom 2. Februar 2011 - BVerwG 6 B 37.10 - Buchholz
421.2 Hochschulrecht Nr. 173). Diese Voraussetzungen liegen bei den von den Klägern aufgeworfenen Beweisfragen entweder nicht vor oder sind nicht ausreichend dargelegt ( $§ 133 \mathrm{Abs}$. 3 Satz 3 VwGO).

a) Das Verwaltungsgericht hat im vorliegenden Rechtsstreit um die Rückgabe eines Bildes nach $\S 5$ Abs. 1 Satz 1 AusglLeistG den Klägern die materielle Beweislast dafür auferlegt, dass diese Bilder bei der besatzungshoheitlichen Enteignung im September 1945 noch Einrichtungsgegenstände des Rittergutes gewesen und nicht schon vorher abhandengekommen sind. Die Kläger halten es für grundsätzlich klärungsbedürftig, ob das Verwaltungsgericht ihre unverschuldete Beweisnot in Bezug auf diese Frage nach dem zum Häftlings-, Vertriebenenund Asylrecht entwickelten Grundsätzen, nach den im Versicherungsrecht für den Fall des Diebstahls entwickelten Prinzipien oder durch eine Reduzierung des Beweismaßes hätte Rechnung tragen müssen (S. 8 und 10 der Beschwerdebegründung).

Diese Fragen bedürfen jedoch keiner rechtsgrundsätzlichen Klärung. Das Bundesverwaltungsgericht hat in Bezug auf vermögensrechtliche Rückgabeansprüche bereits mehrfach entschieden, dass auch im Vermögensrecht die Unerweislichkeit von Tatsachen, aus denen eine Partei ihr günstige Rechtsfolgen herleitet, grundsätzlich zu ihren Lasten geht (Urteil vom 24. März 1994 - BVerwG 7 C 11.93 - BVerwGE 95, 289 [294]; Beschluss vom 17. Mai 2005 - BVerwG 7 B 140.04 - [...] Rn. 6). In bestimmten typischen Sachverhaltskonstellationen - etwa bei Veräußerungen 
im Zusammenhang mit einer Ausreise aus der Deutschen Demokratischen Republik - ist der Beweisnot der Geschädigten durch die Anerkennung eines Anscheinsbeweises Rechnung zu tragen (Urteil vom 26. September 1996 - BVerwG 7 C 14.95 - Buchholz 428 § 1 VermG Nr. 88 und vom 10. März 2009 - BVerwG 8 B 102.08 - [...] Rn. 7). Ob und inwieweit darüber hinaus mit Blick auf einzelne Entschädigungstatbestände des § 1 VermG eine Umkehr der Beweislast oder Beweiserleichterungen in Betracht kommen, lässt sich nicht abstrakt, sondern nur unter Berücksichtigung der konkreten Umstände des Einzelfalls beantworten (Beschluss vom 1. November 1993 - BVerwG 7 B 190.93 - Buchholz 112 § 1 VermG Nr. 11). Dass diese für die vermögensrechtlichen Restitutionsansprüche entwickelten Grundsätze in gleicher Weise auch für den Rückgabeanspruch nach $\S 5$ Abs. 1 AusglLeistG heranzuziehen sind, wird von den Klägern nicht bestritten, liegt auf der Hand und bedarf deshalb keiner Klärung in einem Revisionsverfahren. Die Kläger zeigen auch nicht auf, worin hinsichtlich der Beweisnot der Betroffenen ein zusätzlicher fallübergreifender Klärungsbedarf bestehen soll.

Davon abgesehen verhelfen die hier in Rede stehenden Fragen von angeblich grundsätzlicher Bedeutung der Beschwerde auch deshalb nicht zum Erfolg, weil sie insoweit nicht den Begründungsanforderungen genügt. Soweit die Kläger eine Heranziehung der im Häftlings-, Vertriebenen- und Asylrecht entwickelten Beweiserleichterungen befürworten, wird deren Entscheidungserheblichkeit für den vorliegenden Fall nicht überzeugend dargelegt. Zwar wird im Häftlings-, Vertriebenenund Asylrecht den Einlassungen des Betroffenen besonderes Gewicht beigemessen, weil diese als "Zeugen in eigener Sache" zumeist das einzige Beweismittel sind (Urteil vom 9. Dezember 2010 - BVerwG 10 C 13.09 - BVerwGE 138, 289 Rn. 19 - Buchholz 310 § 130 a VwGO Nr. 82). Die Kläger können jedoch nicht aufgrund eigenen Erlebens "bezeugen", dass das streitgegenständliche Bild noch im Zeitpunkt der Enteignung zum Inventar des Rittergutes gehört hat.

Ebenso wenig würde eine Heranziehung der im Versicherungsrecht für das Vorliegen eines Diebstahls entwickelten Beweiserleichterungsgrundsätze weiterhelfen. Nach der Rechtsprechung des Bundesgerichtshofs genügt es zwar, wenn der Versicherungsnehmer einen Sachverhalt nachweist, der nach der Lebenserfahrung mit hinreichender Wahrscheinlichkeit den Schluss auf eine bedingungsgemäß versicherte Entwendung zulässt (st. Rspr., BGH Urteile vom 5. Oktober 1983 - IVa ZR 19/82 - VersR 1984, 29, und vom 13. März 1993 - IV ZR 74/90 - NJW 1991, 3284). Zu den vom Versicherungsnehmer in vollem Umfang nachzuweisenden Tatsachen, die das äußere Bild einer Entwendung ausmachen, gehört jedoch, dass die als gestohlen gemeldete Sache vor dem behaupteten Diebstahl am angegebenen Ort vorhanden war (BGH Urteile vom 18. Oktober 2006 - IV ZR 130/05 - NJW 2007, 372, und vom 14. Juni 1995 - IV ZR 116/94 - VersR 1995, 956). Demnach wäre die hier interessierende Frage, ob das streitgegenständliche Bild zum Zeitpunkt der Enteignung am angegebenen Ort als Einrichtungsgegenstand vorhanden war, gerade nicht von der Beweiserleichterung er- fasst. Für die geforderte Reduzierung des Beweismaßes wäre kein Raum, weil das Verwaltungsgericht keine „überwiegende Wahrscheinlichkeit" für die behauptete Inventarenteignung festgestellt hat.

b) Soweit die Kläger in vier weiteren Grundsatzrügen (S. 11 bis 14 der Beschwerdebegründung) die Beweiseignung des für die Kunstgegenstände aufgestellten Sammlungskatalogs, die Bedeutung der Aufnahme des Gemäldes in die Sammlung für einen Anscheinsbeweis und die Frage der Erschütterung des Anscheinsbeweises problematisieren, wird die grundsätzliche Klärungsbedürftigkeit und Entscheidungserheblichkeit dieser Fragen nicht in der von $\S 133$ Abs. 3 Satz 3 VwGO geforderten Weise dargelegt. Es wird nicht ausgeführt, weswegen die vom Verwaltungsgericht nicht in Zweifel gezogene Eignung des handschriftlichen Katalogs als Beweisurkunde einer höchstrichterlichen Klärung bedürfte. Ebenso wenig wird erläutert, aus welchen Gründen es im vorliegenden Fall oder in einer Vielzahl von anderen Verfahren auf die Frage ankommen kann, ob das „allgemeine Lebensrisiko ... die grundsätzliche Beweiseignung eines Beweismittels in zeitlicher Hinsicht ... beschränken“ könne. Das Verwaltungsgericht hat jedenfalls bei der Annahme, dass der Katalog nur den Bestand der Sammlung bis Kriegsende widerspiegelt, allein darauf abgestellt, dass der Katalog danach nicht fortgeführt worden ist.

Nicht erläutert wird schließlich, aus welchen Gründen ein den Einzelfall übersteigender Klärungsbedarf zu den Grundsätzen des Anscheinsbeweises bestehen soll. Da die allgemeinen Voraussetzungen des Anscheinsbeweises höchstrichterlich geklärt sind (vgl. etwa Beschluss vom 18. November 1999 - BVerwG 7 B 136.99 - Buchholz 428 § 1 Abs. 3 VermG Nr. 8), hätte zur Begründung der Grundsatzrüge dargelegt werden müssen, aus welchen Gründen eine Ergänzung, Konkretisierung oder Modifizierung dieser Grundsätze für eine größere Zahl von Fällen geboten sein soll. Daran fehlt es. Die Beschwerdeschrift versucht lediglich einzelfallbezogen, der Feststellung des Verwaltungsgerichts auszuweichen, dass es hinsichtlich des Schicksals von Kunst- und Einrichtungsgegenständen in thüringischen Landgütern nach Kriegsende keine verallgemeinerungsfähigen Geschehensabläufe gegeben hat und dass daher die Voraussetzungen für einen Anscheinsbeweis nicht vorliegen.

2. Keinen Erfolg hat auch die zum Prüfungsumfang bei Drittwidersprüchen erhobene Grundsatzrüge. Die von den Klägern gestellte Frage ist bereits höchstrichterlich geklärt. Nach der Rechtsprechung des Bundesverwaltungsgerichts kann ein Drittwiderspruch nur im Falle einer subjektiven Rechtsverletzung des Dritten Erfolg haben. Anderenfalls muss ein Verwaltungsgericht den mangels Rechtsverletzung des Dritten rechtswidrigen Widerspruchsbescheid aufheben (Urteile vom 18. Mai 1982 - BVerwG 7 C 42.80 - BVerwGE 65, 313 [318 f.] = Buchholz $406.25 \S 5$ BImSchG Nr. 3, und vom 15. Juli 1987 - BVerwG 4 C 56.83 - Buchholz 406.19 Nachbarschutz Nr. 73 - [...] Rn. 22). Es kann dahinstehen, ob das angegriffene Urteil dieser Rechtsprechung Rechnung trägt. Mit einer unterlassenen oder unrichti- 
gen Anwendung des Rechts kann eine rechtsgrundsätzliche Bedeutung einer Sache nicht begründet werden (Beschluss vom 27. Januar 2010 - BVerwG 5 B 11.09 - [...] Rn. 6).

3. Schließlich liegen auch die Voraussetzungen für eine Zulassung der Revision nach § 132 Abs. 2 Nr. 3 VwGO nicht vor. Zwar kann eine mit der Verfahrensrüge angreifbare Verletzung des Überzeugungsgrundsatzes nach § 108 Abs. 1 Satz 1 VwGO vorliegen, wenn eine im Einzelfall willkürliche Sachverhalts- und Beweiswürdigung - etwa in Form widersprüchlicher oder aktenwidriger Feststellungen oder infolge von Verstößen gegen Denkgesetze - gegeben ist (Beschlüsse vom 27. Februar 2007 - BVerwG 6 B 81.06 - Buchholz 402.41 Allgemeines Polizeirecht Nr. 83, und vom 1. Juni 2010 - BVerwG 6 B 77.09 - [...] Rn. 14 $\mathrm{mwN})$. Das Verwaltungsgericht hat jedoch in der angegriffenen
Urteilspassage (UA S. 15/16) widerspruchsfrei ausgeführt, dass es für das Schicksal von Einrichtungs- und Kunstgegenständen in den ersten Nachkriegsmonaten keinen typischen Geschehensablauf gebe. In diesen Ausführungen ist weder eine willkürliche Würdigung des Sachverhalts noch ein Verstoß gegen Denkgesetze zu erkennen.

4. Von einer weiteren Begründung wird abgesehen ( $§ 133$ Abs. 5 Satz 2 Halbs. 2 VwGO).

5. Die Kostenentscheidung folgt aus § 154 Abs. 2 VwGO. Die außergerichtlichen Kosten der Beigeladenen sind mangels Beteiligung nach § 162 Abs. 3 VwGO nicht zu erstatten. Die Festsetzung des Streitwerts beruht auf $\S 47$ Abs. 1 und 3, § 52 Abs. 1 GKG. (Entscheidung von der Redaktion bearbeitet.)

\section{„Heatball“ als Kunstobjekt?}

OVG Nordrhein-Westfalen, Beschluss vom 24. Februar 2012 - 4 B 978/11

\section{Über das Verbot des Inverkehrbringens von eingeführten sog. Heatballs, die Einstufung von „Heatballs“ als Kunstobjekte und das Verbot bestimmter energieintensiver Glühlampen. (Leitsatz der Redaktion)}

\section{Tenor}

- Die Beschwerde wird zurückgewiesen.

Die Antragstellerin trägt die Kosten des Beschwerdeverfahrens.

Der Streitwert wird auch für das Beschwerdeverfahren auf 33.800,- Euro festgesetzt.

\section{Gründe}

Die Beschwerde hat keinen Erfolg.

Die von der Antragstellerin fristgerecht dargelegten Gründe, auf deren Prüfung der Senat nach § 146 Abs. 4 S. 1 und 6 VwGO beschränkt ist, führen zu keiner Änderung der angefochtenen Entscheidung.

Das Verwaltungsgericht ist zu Recht davon ausgegangen, dass sich das von der Antragsgegnerin ausgesprochene Verbot des Inverkehrbringens der von der Antragstellerin eingeführten sog. Heatballs voraussichtlich als rechtmäßig erweisen wird.

\section{1.}

Als Ermächtigungsgrundlage kann sich die Antragsgegnerin auf $\S 7$ des Gesetzes über die umweltgerechte Gestaltung energieverbrauchsrelevanter Produkte (EVPG) in der Fassung des 1. Änderungsgesetzes zum Gesetz über die umweltgerechte Gestaltung energiebetriebener Produkte (EBPG) vom 16. November 2011 berufen. Nach dem unverändert gebliebenen § 7 Abs. 3
Satz 1 EVPG trifft die Behörde die erforderlichen Maßnahmen, um die Einhaltung der Rechtskonformität energieverbrauchsrelevanter Produkte ( $\$ 4$ EVPG) zu gewährleisten. Hierzu gehört gemäß § 7 Abs. 3 Satz 2 Nr. 6 EVPG insbesondere die Befugnis, dem Betroffenen zu verbieten, ein solches Produkt in den Verkehr zu bringen, wenn es den Anforderungen des § 4 EVPG nicht genügt.

Durch diese Regelungen setzt der nationale Gesetzgeber - insoweit ohne über einen Umsetzungsspielraum zu verfügen die Richtlinie 2005/32/EG des Europäischen Parlaments und des Rates vom 6. Juli 2005 zur Schaffung eines Rahmens für die Festlegung von Anforderungen an die umweltgerechte Gestaltung energiebetriebener Produkte (Amtsblatt EG L-191, 29) um. Nach Art. 3 der Richtlinie ergreifen die Mitgliedstaaten die erforderlichen Maßnahmen um sicherzustellen, dass energiebetriebene Produkte nur in Verkehr gebracht und/oder in Betrieb genommen werden, wenn sie den für sie geltenden Durchführungsmaßnahmen entsprechen und die CE-Kennzeichnung gemäß Art. 5 tragen.

Dementsprechend verfügen die Mitgliedstaaten weder bei der gesetzlichen Umsetzung der Richtlinie noch bei Anwendung des Gesetzes über einen Entscheidungsspielraum, der es erlaubte, von erforderlichen Maßnahmen zur Durchsetzung von in Durchführungsvorschriften enthaltenen Verboten im Einzelfall abzusehen (vgl. zur entsprechenden Regelungssystematik des Gerätesicherheitsgesetzes Kindt VersR 2004, 296; aA offenbar Scheel in: Landmann/Rohmer, Gewerberecht [Lo- 